ARTICLE OPEN

Check for updates

\title{
Alcohol-abuse drug disulfiram targets pediatric glioma via MLL degradation
}

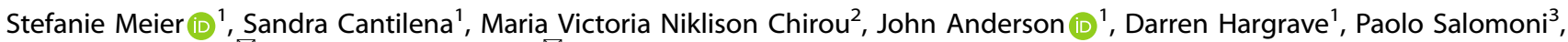 \\ Jasper de Boer (1) ${ }^{1 凶}$ and David Michod (10 $)^{1 凶}$
}

(c) The Author(s) 2021

Pediatric gliomas comprise a broad range of brain tumors derived from glial cells. While high-grade gliomas are often resistant to therapy and associated with a poor outcome, children with low-grade gliomas face a better prognosis. However, the treatment of low-grade gliomas is often associated with severe long-term adverse effects. This shows that there is a strong need for improved treatment approaches. Here, we highlight the potential for repurposing disulfiram to treat pediatric gliomas. Disulfiram is a drug used to support the treatment of chronic alcoholism and was found to be effective against diverse cancer types in preclinical studies. Our results show that disulfiram efficiently kills pediatric glioma cell lines as well as patient-derived glioma stem cells. We propose a novel mechanism of action to explain disulfiram's anti-oncogenic activities by providing evidence that disulfiram induces the degradation of the oncoprotein MLL. Our results further reveal that disulfiram treatment and MLL downregulation induce similar responses at the level of histone modifications and gene expression, further strengthening that MLL is a key target of the drug and explaining its anti-oncogenic properties.

Cell Death and Disease (2021)12:785; https://doi.org/10.1038/s41419-021-04078-9

\section{INTRODUCTION}

Aberrant epigenetic landscapes are prevalent in cancer cells and contribute strongly to cancer development and maintenance. The idea of epigenetic therapy is to interfere with the epigenetic machinery in cancer cells to reverse aberrant patterns and to reestablish the epigenetic landscapes of healthy cells. Thereby, gene expression is normalized, and control mechanisms suppressed in malignant cells, such as cell cycle regulation or apoptosis, are restored [1]. MLL1 and MLL2 are the human homologs of Trithorax (Trx), an epigenetic regulator in Drosophila. The methylation of histone $\mathrm{H} 3$ lysine 4 (H3K4) residues by Trx leads to the maintenance of gene expression. The methyltransferases play a crucial role in the regulation of multiple processes during development and are implicated in the maintenance of Hox cluster gene expression [2]. MLL1 was found to enable cancer stem cell features and to promote cell growth and tumorigenicity in adult glioblastoma [3,4]. MLL2 mutations were identified in $14 \%$ of Medulloblastoma patients [5]. Further, aberrant expression of multiple Hox cluster genes was detected in gliomas [6-8], and a Hox signature was associated with resistance to the chemotherapeutic agent temozolomide in pediatric glioblastoma [9].

The process of traditional drug development is not only long and costly but also inefficient. Only around $5 \%$ of cancer drugs that enter clinical trials end up being successful [10]. Since the market for drugs for rare diseases, such as pediatric cancers, is small, their development can therefore be commercially unattractive. Drug repurposing - the use of a drug for a different indication than it was originally designed and approved for-is a way to tackle the problems associated with traditional drug development [11]. The alcohol abuse drug disulfiram (Antabuse ${ }^{\mathrm{TM}}$ ) has become of interest for drug repurposing due to its preclinically described anti-oncogenic properties against various human cancers [12]. Epidemiological studies revealed a trend towards reduced risk of death from cancer for patients using disulfiram as an anti-alcoholic treatment [13]. Ongoing clinical studies and literature point to the efficacy of disulfiram as a standalone or in combination with other drugs against metastatic liver cancer, lung cancer, prostate cancer, glioblastoma, and melanoma (http://clinicaltrials.gov).

In this report, we show that disulfiram efficiently inhibits cell proliferation of pediatric glioma cell lines and patient-derived primary cells. In addition, we propose disulfiram-induced MLL degradation as a novel mechanism of action for its cytotoxic effects in pediatric gliomas.

\section{RESULTS}

\section{MLL downregulation inhibits cell proliferation}

Gallo and colleagues [3] provided strong evidence in support of a functional MLL-HOXA10 axis in adult glioma stem-like cells. Since HOXA9 and HOXA10 have been associated with temozolomide resistance in pediatric glioma [9], we hypothesized that MLL proteins might also have a functional role in pediatric glioma. We first studied the expression levels of the two MLL proteins in five

\footnotetext{
${ }^{1}$ Cancer Section, Development Biology and Cancer Programme, UCL Great Ormond Street Institute of Child Health, London, UK. ${ }^{2}$ Centre for Therapeutic Innovation, Department of Pharmacy \& Pharmacology, University of Bath, Bath, UK. ${ }^{3}$ Nuclear Function in CNS Pathophysiology, German Center for Neurodegenerative Diseases, Bonn, Germany. ${ }^{\varpi}$ email: j. boer@ucl.ac.uk; d.michod@ucl.ac.uk

Edited by Dr. Angelo Peschiaroli
}

Received: 17 February 2021 Revised: 19 July 2021 Accepted: 20 July 2021

Published online: 11 August 2021 
A

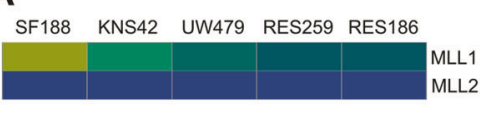

Not detectable Highly expressed

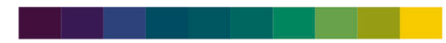

C

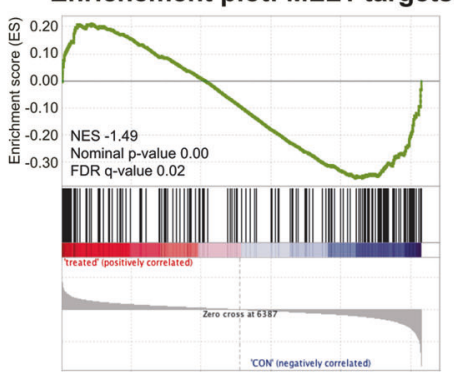

$\mathbf{F}$
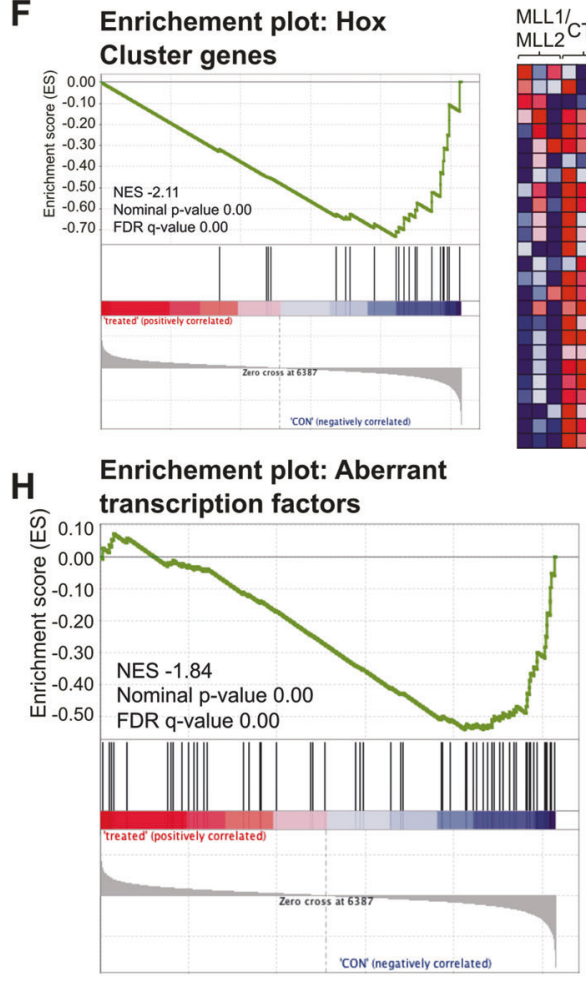

B

CTRL siRNA $>$ MLL1/MLL2 siRNA

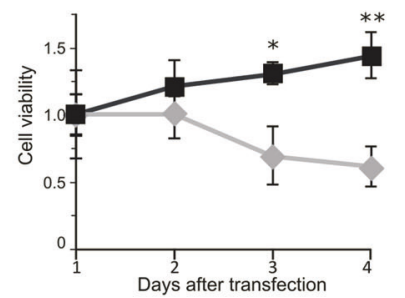

D

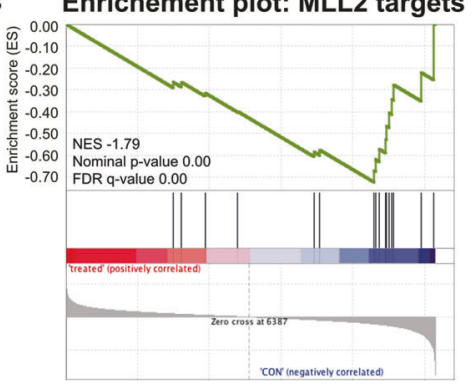

E Enrichement plot: Genes

mediating apoptosis

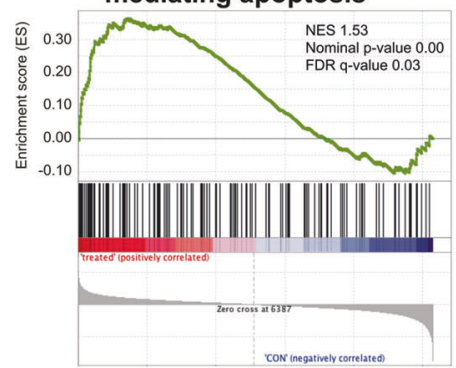

$$
\text { SiRNA }
$$

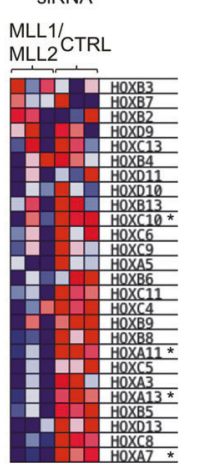

G

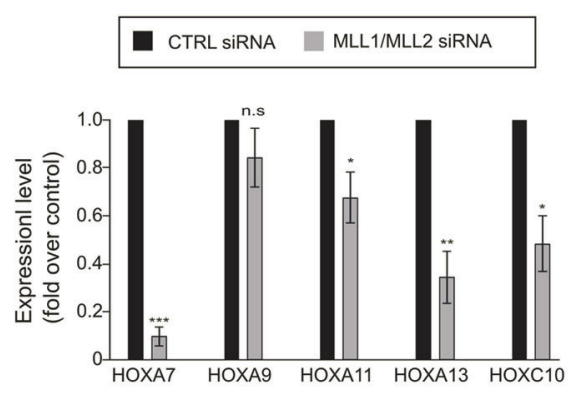

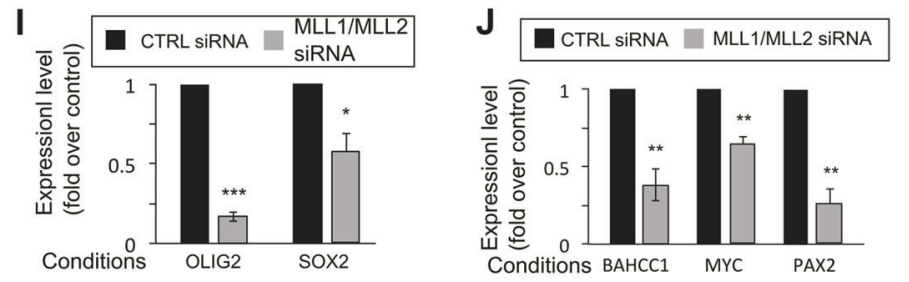

K

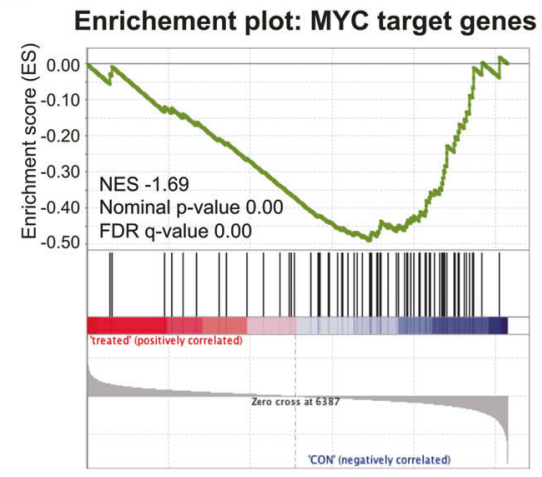

well-characterized pediatric glioma cell lines by analyzing previously published gene expression data (Supplementary Fig. 1A) [14]. Expression of MLL2 is consistent in all five cell lines, while MLL1 expression differs widely over the different cells lines with the highest expression being detected for the high-grade SF188 cells (Fig. 1A). We confirmed this data by western blot analysis (Supplementary Fig. 1b, MLL2 was not detectable by western blot analysis in SF188 cells with different commercially available antibodies). To explore the functional roles of MLL proteins, we downregulated MLL1 and MLL2 in SF188 cells using a pool of 
Fig. 1 MLL downregulation inhibits cell proliferation and decreases H3K4 methylation. A Array data analysis derived from Bax et al. [14]. In the heat map, the relative expression levels of MLL1 and MLL2 are shown. B SF188 cells were seeded in six-well plates at a density of $0.4 \times 10^{6}$ cells/well and transfected with 25 pmol ON-TARGETplus Control pool Non-Targeting pool (labeled C), or ON-TARGETplus SMART pool siRNA targeting MLL1 and MLL2 (labeled siRNA). Cell viability assays were performed on the indicated days. Data are mean \pm STD; $n=3 ;{ }^{*} p<0.05,{ }^{* *} p$ $<0.01$; student $t$-test. C GSEA showing significant negative enrichment of the WANG_MLL_TARGETS gene set after MLL1/MLL2 downregulation in SF188 cells. The gene set comprises genes that require MLL1 for H3K4me3 and expression in mouse embryonic fibroblast cells. D GSEA showing significant negative enrichment of the MLL2_TARGETS gene set for MLL1/MLL2 downregulation in SF188. The gene set comprises previously described MLL2 targets (see material and method). E GSEA showing significant negative enrichment of genes that mediate apoptosis by activation of caspases (MSigDB gene set: HALLMARK_APOPTOSIS) for MLL1/MLL2 downregulation in SF188 cells. F GSEA showing significant negative enrichment of Hox cluster genes for MLL1/MLL2 downregulation in SF188 cells. The gene set comprises all Hox cluster genes. The heatmap represents Hox cluster genes expression in the three independent experiments. Genes present in the core enrichment signature are highlighted in light green, genes marked with an asterisk were further analyzed by qPCR. G: qPCR analyses of selected HOX genes expression. Data are mean \pm STD; $n=3 ;{ }^{*} p<0.05,{ }^{* *} p<0.01$; one-sample $t$-test. H GSEA showing significant negative enrichment of transcription factors with aberrant expression in glioblastoma as reported by Rheinbay et al. [20]. I and J qPCR analyses of selected transcription factors expression upon MLL1/MLL2 downregulation. Data are mean \pm STD; $n=3 ;{ }^{*} p<0.05,{ }^{* * *} p<0.01,{ }^{* * * *} p<0.001 ;$ one-sample $t$ test. k GSEA showing downregulation of MYC targets genes set for MLL1/MLL2 downregulation in SF188 cells.

commercial siRNAs (Supplementary Fig. 1c, d). In contrast to the double downregulation, downregulation of either MLL1 or MLL2 alone did not lead to a significant reduction of H3K4 methylation compared with cells transfected with control siRNAs (Supplementary Fig. 1e). This indicates a functional redundancy of the two methyltransferases. We next assessed the impact of the MLL double downregulation on cell proliferation and detected a decrease in cell viability (Fig. 1B). Cell viability started to decline 3 days after transfection and reached a decrease of $67 \%$ compared with the control 4 days after transfection. To define the role of MLL1 and MLL2 on gene expression in pediatric glioma, we performed RNA-seq experiments. MLL1 and MLL2 were downregulated and RNA was extracted $72 \mathrm{~h}$ after transfection. As shown in Supplementary Fig. 1f, around 700 genes were downregulated upon MLL1 and MLL2 downregulation, while approximately 450 genes were upregulated. Gene Set Enrichment Analysis (GSEA) revealed that the $M L L$ downregulation data was significantly negatively enriched for previously published target genes of MLL1 and MLL2 (Fig. 1C, D) [15-18]. Since MLL downregulation led to a decrease in cell viability, we next wondered if genes involved in apoptosis were modulated by MLL downregulation. GSEA revealed significant positive enrichment of genes mediating apoptosis showing that MLL1 and MLL2 downregulation leads to increased expression of genes involved in apoptosis (Fig. 1E). To learn more about MLL-regulated targets in SF188 cells, we next considered additional gene sets relevant to MLL. HOX genes are known targets of MLL proteins and are associated with resistance to temozolomide in pediatric glioblastoma [9]. GSEA revealed significant negative enrichment of the HOX gene set upon MLL downregulation in SF188 cells (Fig. 1F). The differential expression of five HOX genes was validated by qPCR (Fig. 1G). Irregular activity of transcription factors can have a major effect on gene expression and is frequently observed in cancer [19]. Rheinbay and co-workers [20] described a network of transcription factors that are aberrantly expressed in glioblastoma and promote the maintenance of cancer stem cells. We built a gene set comprising all 75 transcription factors described in this study and performed a GSEA. We observed a negative enrichment of this gene set upon MLL1 and MLL2 downregulation, which implies that MLL1 and/or MLL2 are involved in the positive regulation of these transcription factors, and thus might contribute to the maintenance of glioma stemness (Fig. 1H). We confirmed this observation by performing qPCR analysis on two of the transcription factors, OLIG2 and SOX2 (Fig. 1I). Both have been previously found to play important roles in glioma [21-23]. Among the genes downregulated after MLL1 and MLL2 downregulation in our RNA-seq data, we detected three additional transcription factors with known roles in cancer: MYC [24], PAX2 [25], and BAHCC1 [26] (data not shown). We confirmed these data by qPCR analysis (Fig. $1 \mathrm{~J}$ ) and further showed by GSEA that genes known to be regulated by MYC were negatively enriched upon MLL1 and MLL2 downregulation (Fig. 1K), indicating that the MYC target gene network is disrupted. Taken all together these data showed that MLL1 and MLL2 are important for glioma survival and that they regulate key pathways and expression of genes that are important for glioma biology.

\section{Disulfiram induces MLL degradation}

Disulfiram has previously shown therapeutic potential for the treatment of various cancers, including glioblastoma [27-29]. When applied in combination with copper ions, disulfiram kills glioblastoma cells at physiologically achievable conditions, while the cell viability of normal cells is not affected [30]. However, the different mechanisms of action explaining the anti-oncogenic properties of disulfiram are still under investigation. We recently performed a drug screening to identify molecules that target MLL1 fusion proteins in leukemia and identified disulfiram as a candidate [31]. We showed that in addition to targeting MLL1 fusion proteins, disulfiram also induced the degradation of endogenous MLL1 in leukemia cells. To systematically study the effect of disulfiram on MLL1 in pediatric low- and high-grade glioma, we conducted a dose-response experiment on five wellcharacterized pediatric glioma cell lines of different grades (Supplementary Fig. 1a) [14]. Disulfiram induced a decrease in MLL1 protein levels in all tested cells, indicating that the findings from the drug screening in leukemia cells are transferable to pediatric glioma cells (Fig. 2A-E, conditions 1-4). Since disulfiram treatment did not affect MLL1 expression (Supplementary Fig. 2A), we concluded that disulfiram modulates MLL1 at the protein level. Disulfiram contains a highly thiol-reactive functional group, which reacts with cysteine residues [32]. Both MLL1 and its paralog MLL2 contain reactive cysteines in their DNA binding CXXC domain ( $C$ is cysteine; $X$ is any other amino acid), which is essential for the proteins' function and association with chromatin [33]. We showed that prior to degradation, MLL1 is displaced from chromatin by disulfiram strengthening the notion that disulfiram acts on the CXXC domain of MLL (Supplementary Fig. 2b, c). We thus concluded that disulfiram was likely to induce the degradation of MLL proteins by oxidizing their CXXC domains. We next reasoned that if disulfiram-induced MLL degradation by oxidizing its CXXC domain, its action can be enhanced by inhibiting the thioredoxin reductase/thioredoxin system, an intracellular mechanism used by cells to recover from cytotoxic protein thiol oxidation [34] (see model in Supplementary Fig. 2d). Auranofin, a thioredoxin reductase inhibitor, has been already proposed to enhance disulfiram's cytotoxic effect in ovarian cancer cells [35]. We showed that auranofin increased disulfiraminduced MLL1 degradation in pediatric glioma cells when used at a concentration of $0.5 \mu \mathrm{M}$ (Fig. $2 \mathrm{~A}-\mathrm{E}$, conditions 6 and 7), while auranofin alone had no effect on MLL1 protein levels (Fig. 2A-E, condition 5). 
A

\section{SF188}

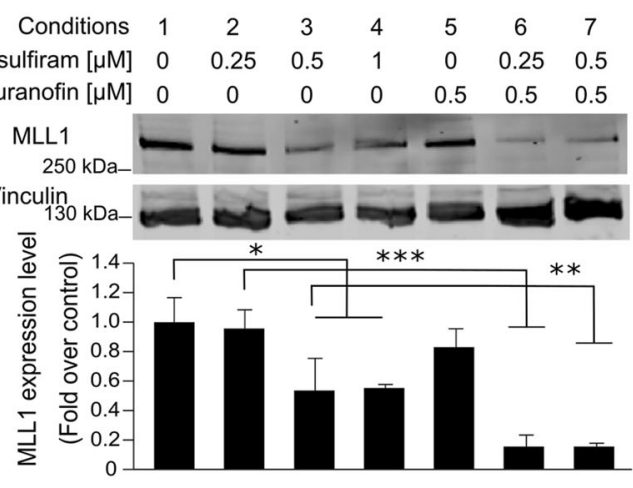

C

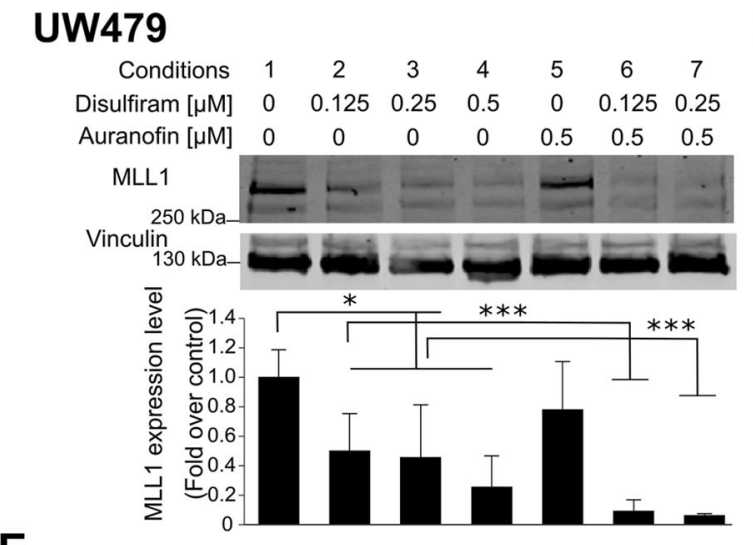

E

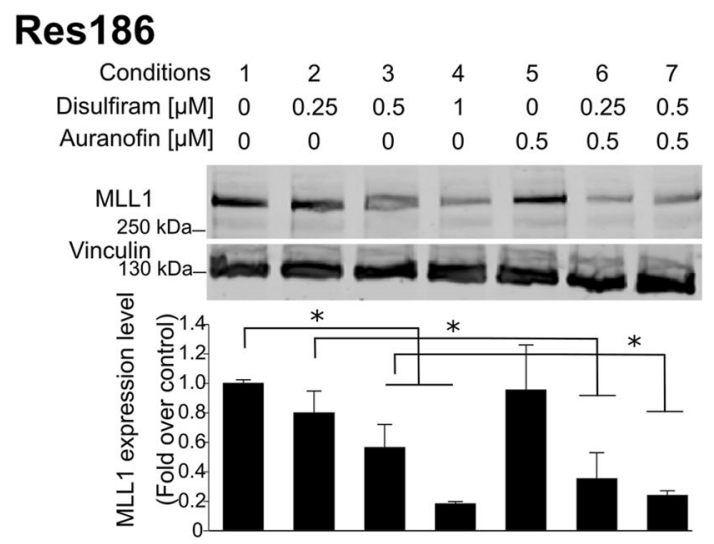

B

\section{KNS42}

\begin{tabular}{|c|c|c|c|c|c|c|c|}
\hline Conditions & 1 & 2 & 3 & 4 & 5 & 6 & 7 \\
\hline sulfiram $[\mu \mathrm{M}]$ & 0 & 0.25 & 0.5 & 1 & 0 & 0.25 & 0.5 \\
\hline
\end{tabular}

$\begin{array}{llllllll}\text { Auranofin }[\mu \mathrm{M}] & 0 & 0 & 0 & 0 & 0.5 & 0.5 & 0.5\end{array}$

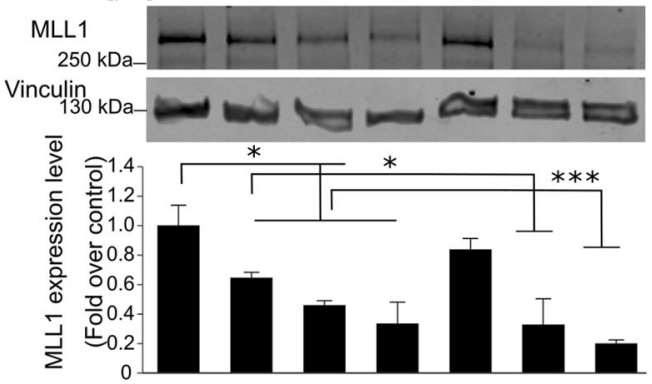

\section{Res259}

$\begin{array}{cccccccc}\text { Conditions } & 1 & 2 & 3 & 4 & 5 & 6 & 7 \\ \text { Disulfiram }[\mu \mathrm{M}] & 0 & 0.062 & 0.125 & 0.25 & 0 & 0.062 & 0.125 \\ \text { Auranofin }[\mu \mathrm{M}] & 0 & 0 & 0 & 0 & 0.5 & 0.5 & 0.5\end{array}$

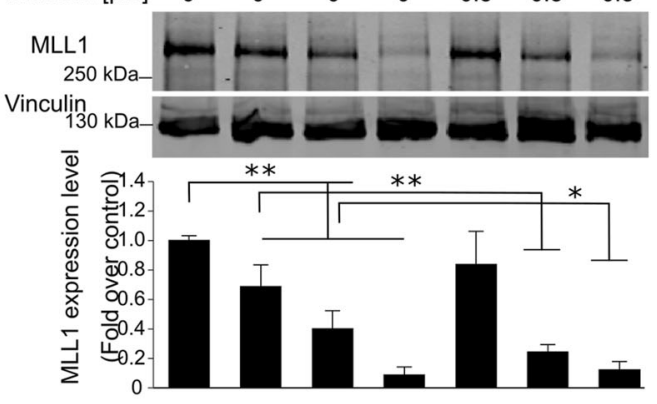

Fig. 2 Disulfiram induces MLL degradation. A-E Top: whole-cell lysates were prepared from the indicated cell lines, cultured in the absence or presence of $0.5 \mathrm{mM}$ auranofin with increasing concentrations of disulfiram for $16 \mathrm{~h}$. Extracts were probed with $\alpha-\mathrm{MLL} 1$ antibody and $\alpha$ vinculin antibody as loading control. Bottom: the graphs represent image analysis of band intensity. Data are mean $\pm \mathrm{STD} ; n=3 ;{ }^{*} p<0.05,{ }^{* * *} p$ $<0.01{ }^{* * *} p<0.001$; two-way analysis of variance (ANOVA) test with Bonferroni posttest.

\section{Disulfiram and auranofin synergistically inhibit cell proliferation of pediatric glioma cell lines}

To systematically study the effect of disulfiram treatment on pediatric low- and high-grade glioma, we conducted time-course and dose-response experiments on the five pediatric glioma cell lines. We monitored the cell viability of treated cells over $48 \mathrm{~h}$ and observed that all pediatric glioma cells were killed within that time frame at disulfiram concentrations lower than $1 \mu \mathrm{M}$ (Fig. 3A-E). Interestingly, we noticed that the half-maximal effective concentrations (EC50s) correlated with the cell lines' grades (Fig. 3A-E). Low-grade glioma cell lines exhibited a lower EC50 and thus higher sensitivity to disulfiram treatment than high-grade cell lines (EC50 of $0.32-0.5 \mu \mathrm{M}$ for Res259 and Res 186, compared with EC50 of $0.62-0.75 \mu \mathrm{M}$ for SF188, KNS42, and UW479). The same doseresponse experiment was performed in combination with three increasing doses of auranofin. As shown in Fig. 3A-E, auranofin itself did not affect cell viability at the concentrations used measured by luminescent cell viability assay, however, it sensitized 
A

\section{SF188}

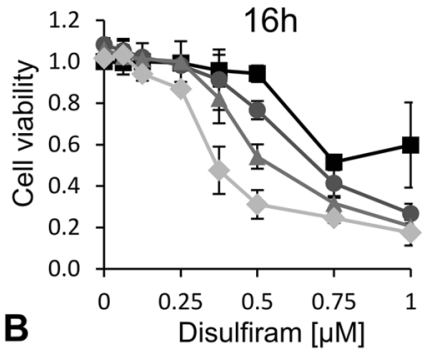

\section{KNS42}

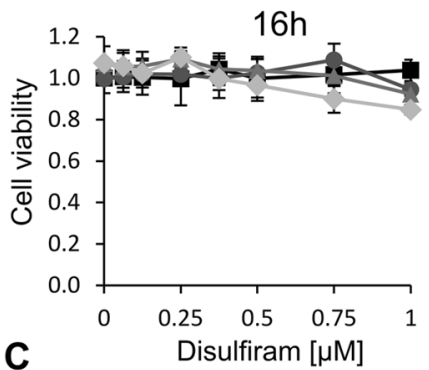

C

UW479

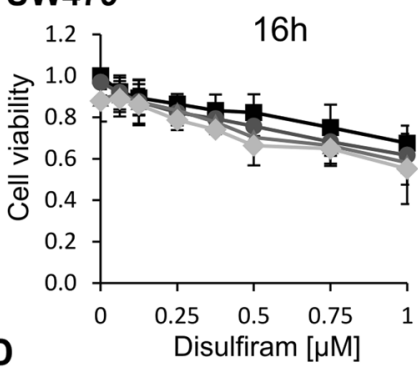

\section{Res259}

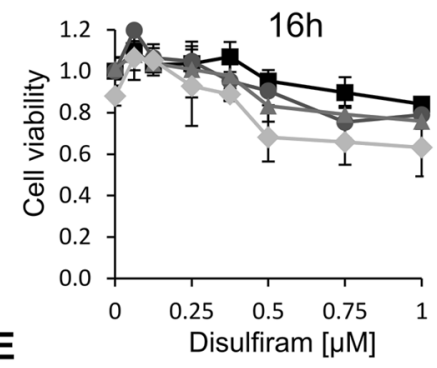

E

Res186

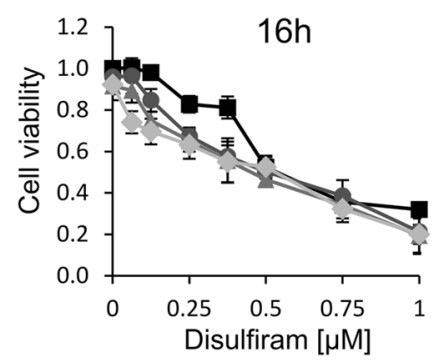

$\begin{array}{ll}\text { Auranofin }[0 \mu \mathrm{M}] & \Delta \text { Auranofin }[0.5 \mu \mathrm{M}] \\ \text { Auranofin }[0.25 \mu \mathrm{M}] & \text { Auranofin }[1 \mu \mathrm{M}]\end{array}$

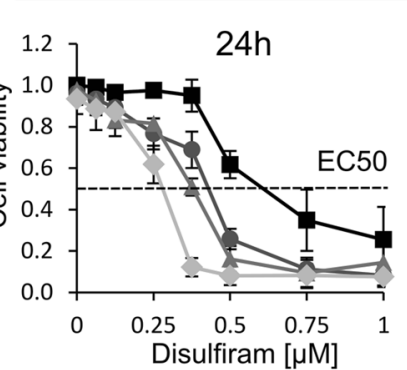

Auranofin $[1 \mu \mathrm{M}]$
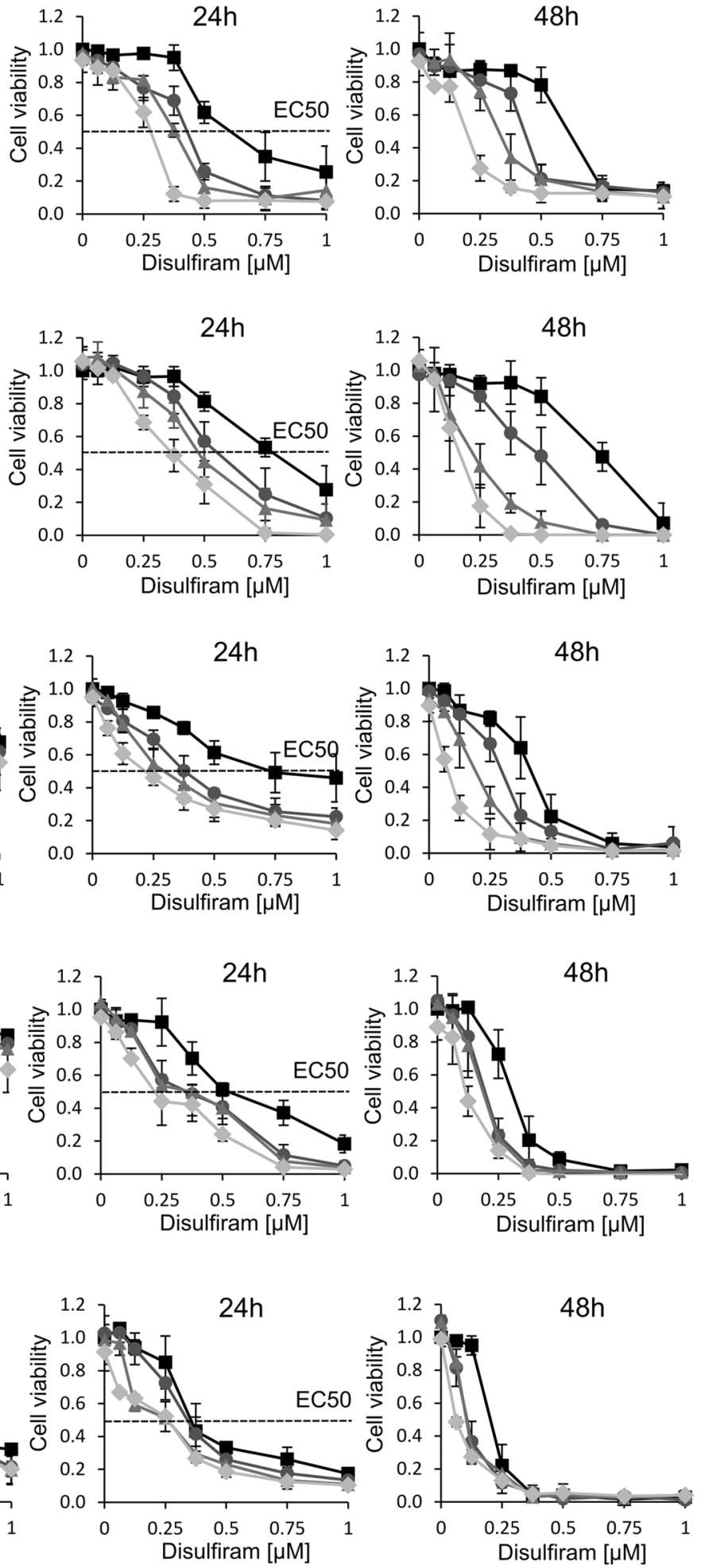

Fig. 3 Disulfiram and auranofin synergistically inhibit cell proliferation. A-E Cells were plated in 96-wells plates. The following day cells were treated with the indicated concentrations of disulfiram in the absence or presence of increasing doses of auranofin. At the indicated time cell viability assays were performed. Data are mean \pm STD; $n=3$. 
A

\section{SF188}

$\begin{array}{llllllll}\text { Conditions } & 1 & 2 & 3 & 4 & 5 & 6 & 7\end{array}$

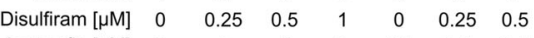

\begin{tabular}{llllllll} 
Auranofin $[\mu \mathrm{M}]$ & 0 & 0 & 0 & 0 & 0.5 & 0.5 & 0.5 \\
\hline
\end{tabular}

$\mathrm{H} 3 \mathrm{~K} 4 \mathrm{me} 3 \longrightarrow-\longrightarrow$

$\mathrm{H} 3 \mathrm{~K} 4 \mathrm{me} 2 \div-\div-\cdots$

$\mathrm{H} 3 \mathrm{~K} 9 \mathrm{me} / 3$
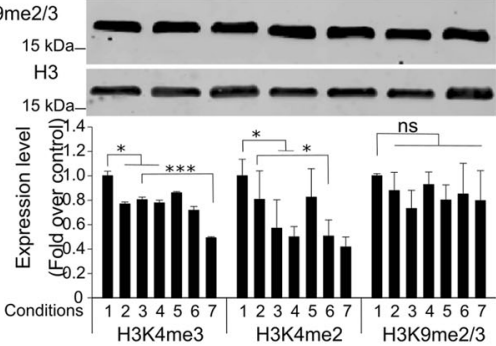

C

UW479

$\begin{array}{llllllll}\text { Conditions } & 1 & 2 & 3 & 4 & 5 & 6 & 7\end{array}$

$\begin{array}{llllllll}\text { Disulfiram }[\mu \mathrm{M}] & 0 & 0.125 & 0.25 & 0.5 & 0 & 0.125 & 0.25\end{array}$

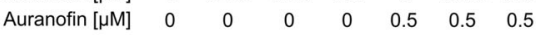

H3K4me3

$\mathrm{H} 3 \mathrm{~K} 4 \mathrm{me} 2$

$15 \mathrm{kDa}$

$\mathrm{H} 3 \mathrm{~K} 9 \mathrm{me} 2 / 3$

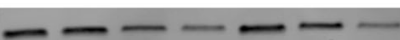

$15 \mathrm{kDa} \cdots-\longrightarrow-\cdots$

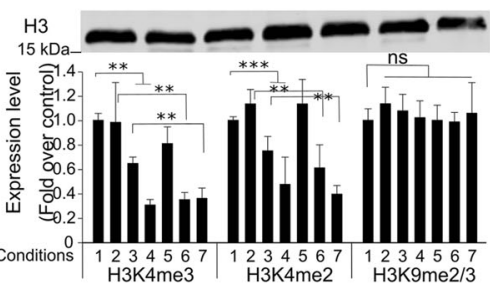

E

\section{Res186}

$\begin{array}{cccccccc}\text { Conditions } & 1 & 2 & 3 & 4 & 5 & 6 & 7 \\ \text { Disulfiram }[\mu \mathrm{M}] & 0 & 0.25 & 0.5 & 1 & 0 & 0.25 & 0.5\end{array}$

Auranofin $[\mu \mathrm{M}] \quad 0 \quad \begin{array}{llllllll}0 & 0 & 0 & 0 & 0.5 & 0.5 & 0.5\end{array}$

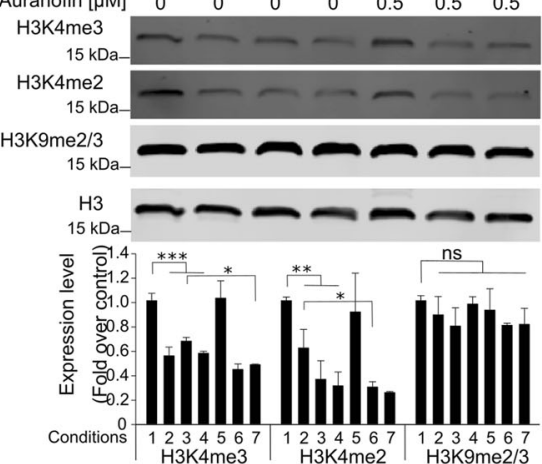

B

\section{KNS42}

$\begin{array}{llllllll}\text { Conditions } & 1 & 2 & 3 & 4 & 5 & 6 & 7\end{array}$

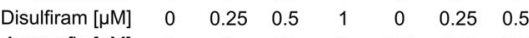

$\begin{array}{llllllll}\text { Auranofin }[\mu \mathrm{M}] & 0 & 0 & 0 & 0 & 0.5 & 0.5 & 0.5\end{array}$

$\mathrm{H} 3 \mathrm{~K} 4 \mathrm{me} 3----$

$15 \mathrm{kDa}-$

H3K4me2 - - - - - - -

$15 \mathrm{kDa}$

$\mathrm{H} 3 \mathrm{~K} 9 \mathrm{me} 2 / 3=-\infty \longrightarrow$

$15 \mathrm{kDa}$

$\mathrm{H} 3$
$15 \mathrm{kDa}-$

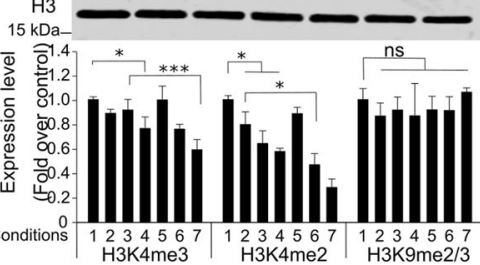

D

Res259

$\begin{array}{llllllll}\text { Conditions } & 1 & 2 & 3 & 4 & 5 & 6 & 7\end{array}$

$\begin{array}{llllllll}\text { Disulfiram }[\mu \mathrm{M}] & 0 & 0.062 & 0.125 & 0.25 & 0 & 0.062 & 0.125\end{array}$

Auranofin $[\mu \mathrm{M}] \quad 0 \quad 0000.5$

$\begin{array}{r}\mathrm{H} 3 \mathrm{~K} 4 \mathrm{me} 3 \\ 15 \mathrm{kDa}-\cdots\end{array}-\frac{1}{-}-\frac{1}{-}$

H3K4me2

$15 \mathrm{kDa}-$

$\mathrm{H} 3 \mathrm{~K} 9 \mathrm{me} 2 / 3$

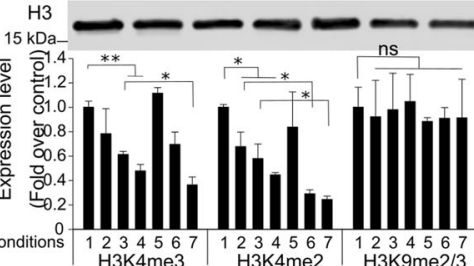

$\mathbf{F}$

\section{ChIP H3K4me2}

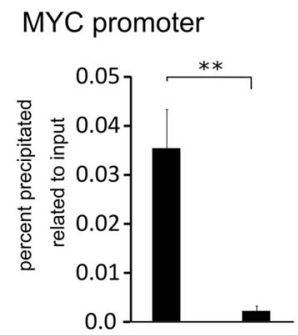

Disulfiram $[\mu \mathrm{M}] \quad 0 \quad 0.5$
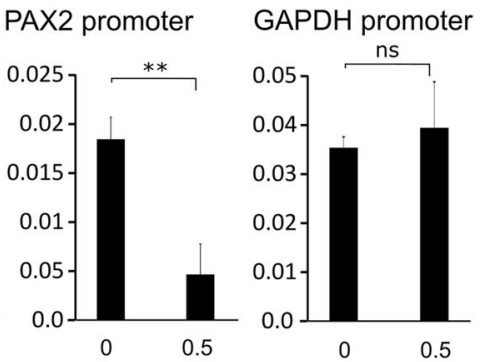

Fig. 4 Disulfiram induces decrease of H3K4 methylation. A-E Top: whole-cell lysates were prepared from the indicated cell lines, cultured in

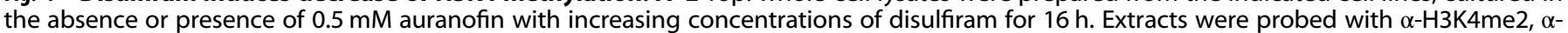

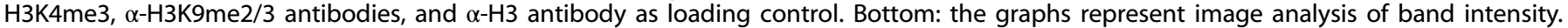

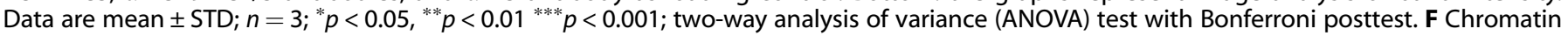

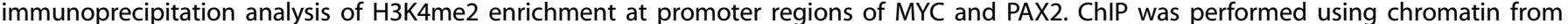
SF188 left untreated or treated for $16 \mathrm{~h}$ with $0.5 \mu \mathrm{M}$ disulfiram. Data are mean $\pm \mathrm{STD} ; n=3{ }^{* *} p<0.01$, student $t$-test.

the cell lines to disulfiram treatment. The combination study proved that the two drugs work synergistically in pediatric glioma (Supplementary Fig. 3a-e). We next showed that disulfiraminduced cell death at $0.5 \mu \mathrm{M}$ and that auranofin itself did not induce cell death but potentiated disulfiram-induced cell death (Supplementary Fig. $3 \mathrm{f}-\mathrm{j}$ ). We further confirmed that disulfiram killed SF188 cells by caspase-3 activation (Supplementary Fig. 3k).

\section{Disulfiram induces histone modifications}

The histone methyltransferases MLL1 and MLL2 are responsible for the addition of methyl groups to the histone $\mathrm{H} 3$ tail amino acid lysine 4 (H3K4) [36]. H3K4 can be mono-, di-, or trimethylated, with both di- and trimethylation being associated with promoter regions of active transcription [37]. To assess whether the treatment with disulfiram alone or in combination with auranofin 
A

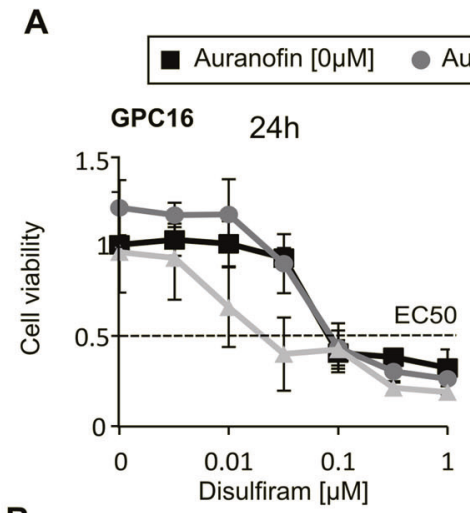

B

$$
\text { GPC16 }
$$

Disulfiram $[\mu \mathrm{M}] \quad 0 \quad 0.032 \quad 0.1 \quad 0.32$
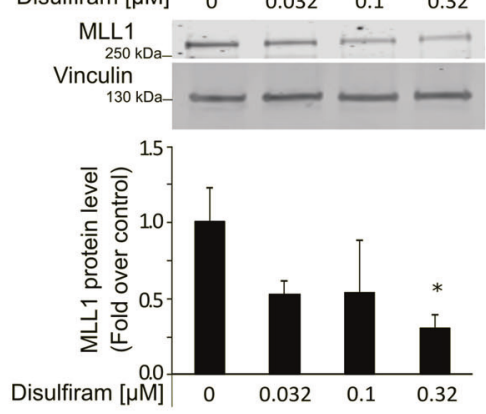

$\triangle$ Auranofin $[0.5 \mu \mathrm{M}]$

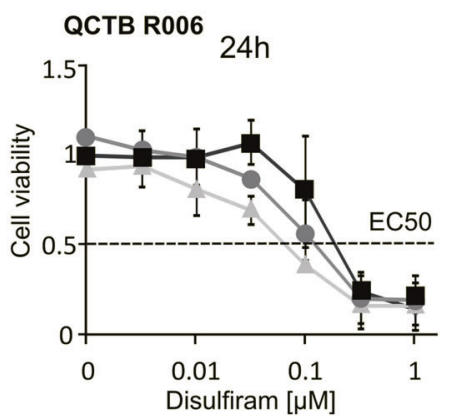

QCTB R006

Disulfiram $[\mu \mathrm{M}] \quad 0 \quad 0.032 \quad 0.1 \quad 0.32$
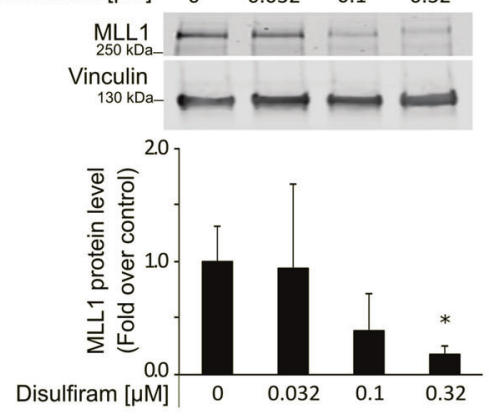

\section{GPC16

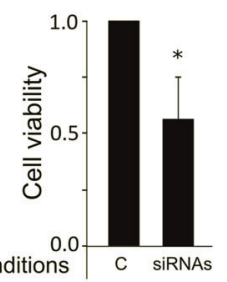

D

GPC16 Enrichement plot: MLL1 targets

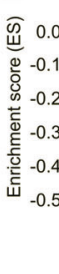

$\mathbf{F}$

\section{GPC16 Enrichement plot: Aberrant} transcription factors

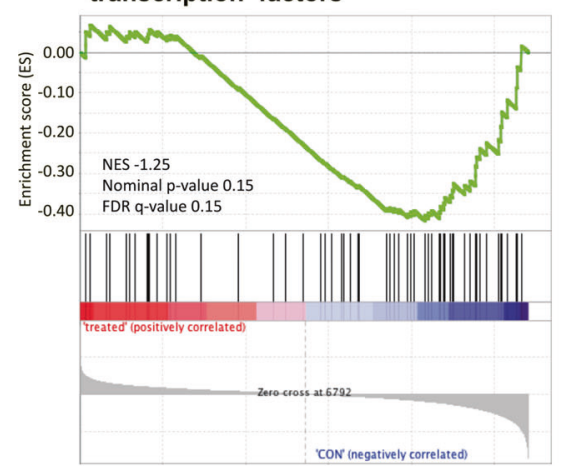

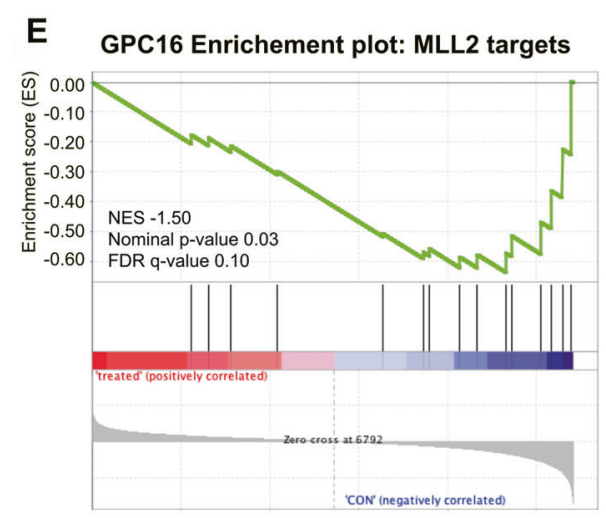

G

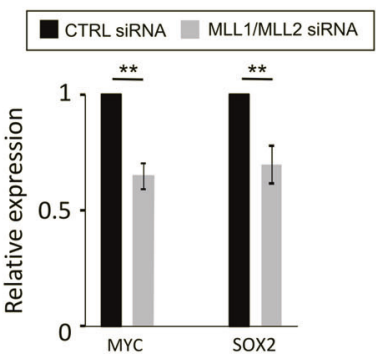

affects the methyltransferase activity, we studied the level of H3K4 methylation in treated cells. As shown in Fig. 4A-E conditions 1-4, disulfiram efficiently decreased the level of H3K4 dimethylation (H3K4me2) in all cell lines, as well as the level of H3K4 trimethylation (H3K4me3), although to a lesser extent. When disulfiram was used in combination with auranofin, the decrease in both di- and trimethylation was even greater (Fig. 4A-E, compare conditions 2 with 6 and conditions 3 with 7), while auranofin alone did not affect the methylation status of H3K4 (Fig. $4 A-E$, condition 5). None of the treatments affected the level of 
Fig. 5 Disulfiram inhibits cell proliferation and induces MLL degradation in patient-derived primary high-grade glioma cells. A Cells were plated in 96-wells plates. The following day cells were treated with the indicated concentrations of disulfiram in the absence or presence of increasing doses of auranofin. At the indicated time cell viability assays were performed. Data are mean \pm STD; $n=3$. B Top: whole-cell lysates were prepared from the indicated cells, cultured in the absence or presence of increasing concentrations of disulfiram for $16 \mathrm{~h}$. Extracts were probed with $\alpha$-MLL1 antibody and $\alpha$-vinculin antibody as loading control. Bottom: the graphs represent image analysis of band intensity. Data are mean \pm STD; $n=3 ;{ }^{*} p<0.05$; two-way analysis of variance (ANOVA) test with Bonferroni posttest. C GPC16 cells were seeded in six-well plates at a density of $0.4 \times 10^{6}$ cells/well and transfected with 25 pmol ON-TARGETplus Control pool Non-Targeting pool (labeled C), or ONTARGETplus SMART pool siRNA targeting MLL1 and MLL2 (labeled siRNA). Cell viability assays were performed 3 days after transfection. Data are mean \pm STD; $n=3$; * $p<0.05$; one-sample $t$-test. D, E, and $\mathbf{F}$ same experiment as Fig. 1 but with GPC16 cells. G qPCR analyses of selected transcription factors expression upon MLL1/MLL2 downregulation in GPC16 cells. Data are mean \pm STD; $n=3 ;{ }^{* *} p<0.01 ;$ one-sample $t$-test.

H3K9 methylation (Fig. 4A-E), indicating that disulfiram specifically interfered with the histone modifications associated with MLL1 and MLL2 activity. We next assessed the level of H3K4 dimethylation upon disulfiram treatment at the promoter region of MYC and PAX2, two genes affected by MLL downregulation (Fig. 1J). We showed that disulfiram-induced a marked decrease of H3K4me2 at both promoter regions of MYC and PAX2 while not affecting the promoter region of GAPDH (Fig. 4F). Thus, indicating that disulfiram-induced a decrease of H3K4 methylation at distinct regions rather than affecting the overall level of H3K4me2.

\section{Disulfiram-induced MLL degradation inhibits proliferation of high-grade glioma patient-derived primary cells}

To confirm our findings in a cell culture model that closely represents the human in vivo patient condition, we used two primary pediatric patient-derived high-grade glioma stem cells, GPC16 and QCTB R006. First, we conducted a disulfiram doseresponse experiment in the absence or presence of increasing doses of auranofin, and we monitored the viability of treated cells over $24 \mathrm{~h}$. We observed that both primary cells were killed within that time frame at disulfiram concentrations lower than $0.1 \mu \mathrm{M}$ for GPC16 and $0.5 \mu \mathrm{M}$ for QCTB R006 (Fig. 5A). In comparison with the high-grade glioma cell lines SF188 and KNS42, the primary cells exhibited lower EC50s (SF188 EC50: $0.62 \mu \mathrm{M}$, KNS42 EC50: $0.75 \mu \mathrm{M}$ (Fig. 2A-E), GPC16 EC50: $0.1 \mu \mathrm{M}$, QCTB R006 EC50: $0.3 \mu \mathrm{M}$ (Fig. 5A)) and thus higher sensitivity to disulfiram. However, the synergistic effect of the disulfiram/auranofin combination treatment on primary cells was not as strong as on high-grade glioma cell lines (synergy scores of 8.4 and 15.1 for GPC16 and QCTB R006 respectively (Supplementary Fig. 4a), compared with 24.5 and 19.5 for SF188 and KNN42 respectively (Supplementary Fig. 3a, b)). The two primary cells also showed a decrease in MLL1 protein levels upon treatment with disulfiram (Fig. 5B). We next tested if downregulation of MLL proteins had a similar effect on cell proliferation as observed for the SF188 cell line. The same siRNA pools were used for the downregulation of MLL1 and MLL2 in the primary cells as previously used in SF188 cells. We achieved a significant downregulation of MLL1 and MLL2 gene expression in GPC16 cells (Supplementary Fig. 4b) that is comparable to what we achieved in SF188 cells (Supplementary Fig 1d). However, the downregulation was not successful in QCTB R006 cells (data not showed), probably due to low transfection efficiency of these cells with the current protocol. We next assessed the effect of MLL1 and MLL2 downregulation on cell proliferation in GPC16. As shown in Fig. 5C, MLL1 and MLL2 downregulation decreased the viability of GPC16 cells within 3 days by $50 \%$. We performed RNA-seq experiments to define the role of MLL1 and MLL2 on gene expression in GPC16 cells and noticed several similarities between the effect of the MLL downregulation in GPC16 and in SF188. As in SF188, it also led to the downregulation of previously published target genes of MLL1 and MLL2 (Fig. 5D, E), as well as genes that are part of the network of transcription factors that are aberrantly expressed in glioblastoma in GPC16 cells (Fig. 5F). We further showed that SOX2 and MYC were also negatively regulated upon MLL1 and MLL2 downregulation, although the downregulations were less marked than in SF188 cells (Fig. 5G). In addition, we also noticed some differences compared with the SF188 cells. HOX genes and PAX2 were not expressed at levels detectable by RNA-seq or qPCR analysis in GPC16 cells (data not shown), indicating that the expression of certain development-related transcription factors may vary between cell lines and primary cells, or generally between glioma cells. Finally, we did not observe an effect of $M L L$ downregulation on the expression of OLIG2 and BAHCC1 (Fig. 5G, H).

Altogether, these results indicate that primary patient-derived cells are more sensitive to disulfiram compared with glioma cell lines. The findings further suggest that disulfiram-induced MLL protein degradation contributes to the inhibition of cell proliferation of primary patient-derived cells. Finally, these data showed that MLL1 and MLL2 generally regulate similar pathways and gene expression in SF188 and GPC16 cells, although there are some differences.

\section{Disulfiram induces transcriptional changes that mimic $M L L$ downregulation}

Our previous experiments showed that disulfiram treatment leads to $M L L$ degradation and a decrease in $\mathrm{H} 3 \mathrm{~K} 4$ methylation. To assess whether disulfiram treatment also affects the expression of genes epigenetically regulated by MLL proteins, we first used RNA-seq to identify genes differentially expressed upon the downregulation of MLL1 and MLL2 with siRNA. Genes identified as significantly downregulated under this condition in both the cell line SF188 and the glioma stem cells GPC16 were used to build a gene set called MLL1_MLL2_core (Supplementary Table 1). Since MLL1 and MLL2 are primarily known to be involved in the positive regulation of gene expression, we focused on genes that showed a decrease in expression upon MLL downregulation. RNA-seq of SF188 and GPC16 cells treated with disulfiram, as well as untreated cells showed that the drug treatment led to differential expression of around 8000 genes (Supplementary Fig. 5a). GSEA revealed significant negative enrichment for the MLL1_MLL2 core genes set in disulfiram-treated SF188 and GPC16 cells, demonstrating that genes regulated by MLL1 and MLL2 are affected by disulfiram treatment in both glioma cell line and primary cells (Fig. 6A). This indicates that the decrease in MLL1 and MLL2 protein levels upon disulfiram treatment, and thus the reduced methyltransferase activity, leads to the downregulation of genes normally positively regulated by the two methyltransferases. To learn more about the effect of disulfiram on MLL-regulated targets, we next considered additional gene sets relevant to MLL. GSEA revealed significant negative enrichment of Hox cluster gene set upon disulfiram treatment in SF188 cells, showing that the treatment leads to the downregulation of Hox cluster genes (Fig. 6B). Their differential expression upon disulfiram treatment was validated by qPCR (Fig. 6C). While HOXA9 expression was not detectable by RNA-seq, it was detectable in disulfiram-treated and untreated cells using qPCR (Fig. 6C). These findings show that disulfiram treatment affects the expression of additional genes regulated by MLL1 and MLL2, and that disulfiram can be used to disrupt the expression of HOX genes, which are frequently associated with tumorigenesis in high-grade glioma [3]. Among the genes downregulated upon MLL1 and MLL2 downregulation, we detected BAHCC1 MYC and PAX2 (Fig. 1J). Using qPCR we confirmed that the three genes were 
A
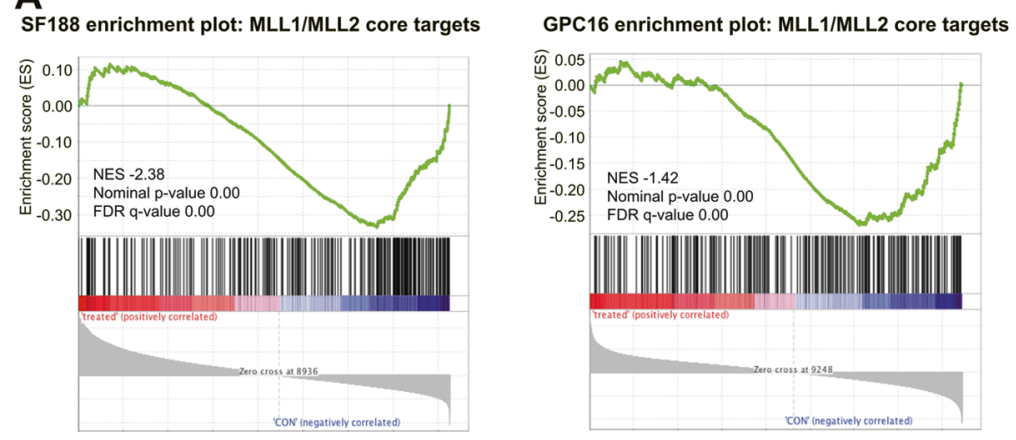

B
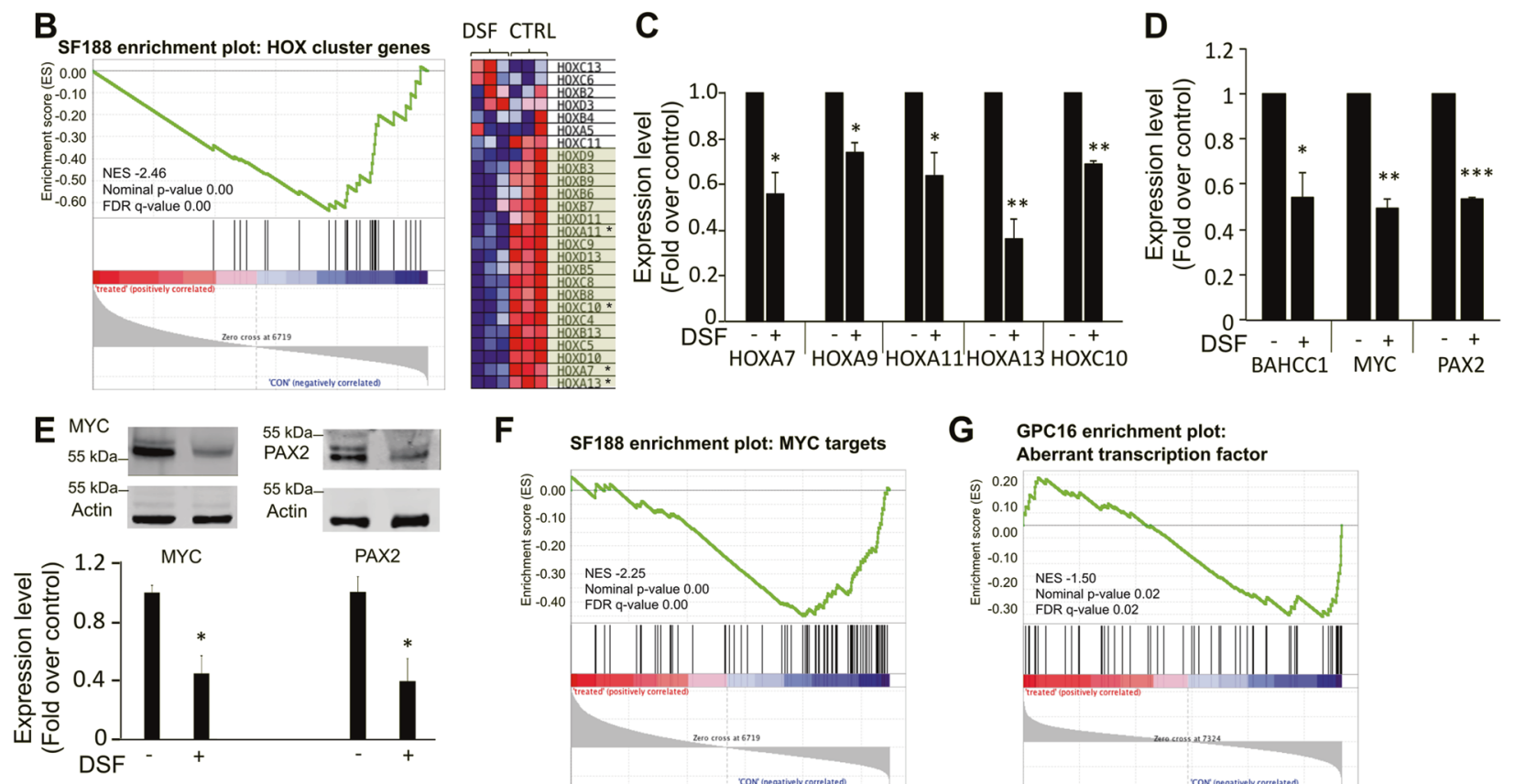

F

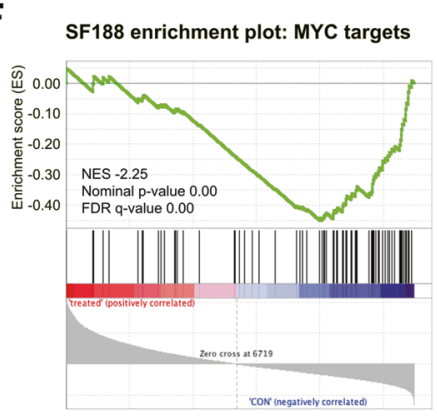

G GPC16 enrichment plot:
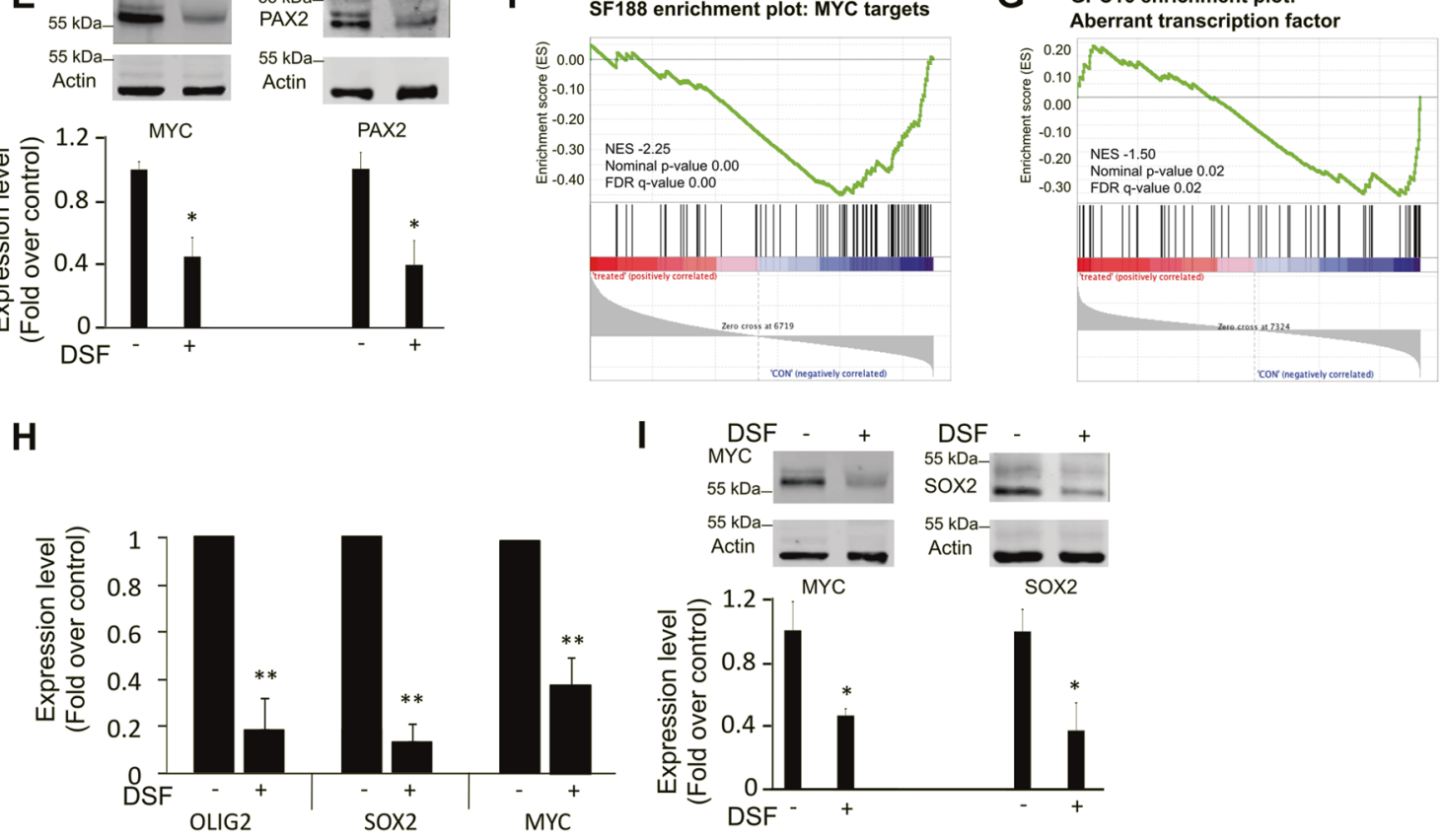

Fig. 6 Changes in SF188 and GPC16 gene expression following disulfiram treatment. A and B GSEA showing downregulation of MLL1/MLL2 core genes set (genes downregulated upon MLL1/MLL2 downregulation) in disulfiram-treated SF188 and GPC16 cells. C GSEA showing downregulation of HOX cluster genes set (gene set including all 39 human Hox cluster genes) in disulfiram-treated SF188 cells. The heatmap is part of the GSEA. It represents Hox cluster genes expression in disulfiram-treated (DSF) and untreated cells (CTRL) with three replicates each. Genes present in the core enrichment signature are highlighted in green, genes marked with an asterisk were further analyzed. C qPCR analyses of HOX genes expression in disulfiram-treated SF188 cells. Data are mean \pm STD; $n=3 ;{ }^{*} p<0.05,{ }^{* *} p<0.01$; one-sample $t$-test. D qPCR analyses of transcription factors expression upon disulfiram treatment. Data are mean \pm STD; $n=3 ;{ }^{*} p<0.05,{ }^{* *} p<0.01,{ }^{* * *} p<0.001 ;$ onesample $t$-test. E Western blot analysis of MYC and PAX2 expression level upon disulfiram treatment. Data are mean \pm STD; $n=3 ;{ }^{*} p<0.05, t-$ test. F GSEA showing downregulation of MYC targets genes set (genes upregulated upon MYC expression) in disulfiram-treated SF188 cells (MSigDB gene set: SCHUHMACHER_MYC_TARGETS_UP). G GSEA showing downregulation of aberrant transcription factor set (75 transcription factors with aberrant expression in glioblastoma as reported by Rheinbay et al.) in disulfiram-treated GPC16 cells. H qPCR analyses of OLIG2, SOX2, and MYC expression in disulfiram-treated GPC16 cells. Data are mean \pm STD; $n=3 ;{ }^{* *} p<0.01$; one-sample $t$-test. I Western blot analysis of SOX2 and MYC expression level upon disulfiram treatment. Data are mean \pm STD; $n=3 ;{ }^{*} p<0.05, t$-test. 
also downregulated in disulfiram-treated SF188 cells (Fig. 6D). We confirmed for MYC and PAX2 that the decrease in gene expression resulted in protein downregulation (Fig. 6E). Further, GSEA revealed a significant negative enrichment of MYC target genes in treated SF188 cells (Fig. 6F), showing that disulfiram treatment not only disrupts the expression of MYC but also leads to the downregulation of its target genes. Similarly, we detected that disulfiram treatment affects the expression of cancer-associated transcription factors in the patient-derived GPC16 cells (Fig. 6G). We further validated this finding by analyzing the expression of some of these transcription factors by $\mathrm{qPCR}$ and western blot. A reduction of over $80 \%, 85 \%$, and $60 \%$ was detected for SOX2, OLIG2, and MYC respectively at the level of gene expression (Fig. $6 \mathrm{H})$, and a reduction of over $50 \%$ was detected for MYC and SOX2 at the protein level (Fig. 6l).

\section{DISCUSSION}

Various mechanisms have been proposed to explain the cytotoxic effect of disulfiram on cancer cells $[29,30,38]$. Disulfiram has been shown to induce proteasome inhibition that leads to the accumulation of misfolded proteins and possible toxic protein aggregates [28]. More recently, disulfiram's tumor-suppressing effects have been attributed to its action on the protein NPL4 and the resulting accumulation of ubiquitinated proteins [13]. We proposed here, that, in addition to the aforementioned targets, disulfiram-induced MLL degradation contributes to its antioncogenic properties. Repurposing of disulfiram for cancer treatment has already been studied in several cancers and multiple clinical trials have been done to validate its potential use as a drug to fight cancer. MLL1 has been associated with many types of cancer [4,39-41]. In addition, MLL1 plays a critical role in tumor growth and angiogenesis and its knockdown suppresses tumor growth in vivo. Our data indicate that disulfiram targets MLL1 [42]. It is thus of interest to further test the efficacy of disulfiram in MLL1-, and potentially MLL2-driven cancers. We showed that disulfiram kills pediatric glioma stem cells at low concentrations and cell lines at slightly higher concentrations. We found that the drug's efficiency can be increased by the addition of auranofin and that the synergistic effect of the two drugs is more pronounced in differentiated cells compared to undifferentiated cells. This indicates that disulfiram alone efficiently kills glioma cancer stem cells and in combination with auranofin can kill more differentiated cancer cells, suggesting that disulfiram is efficient against heterogeneous lineages of cancer cells. In addition to induced MLL1 degradation, disulfiram also interacts with previously proposed targets of the drug in pediatric glioma. This shows that disulfiram's mode of action in pediatric glioma cells is multi-modal and thus advantageous over single target molecules, which are often prone to therapy resistance.

Taken altogether, our study showed that disulfiram kills pediatric glioma cells at doses relevant to human treatment, hence adding pediatric glioma to the list of cancers that potentially can be treated with disulfiram. The current treatment of pediatric is primarily based on chemotherapeutic compounds, and often has serious side effects in children. Well-tolerated drugs such as disulfiram and auranofin are thus of particular interest for the treatment of this disease.

\section{MATERIALS AND METHODS Cell culture}

GPC16 and QCTB ROS 006 were maintained in NeuroCult ${ }^{\text {TM }}$ NS-A Basal medium (Human, STEMCELL ${ }^{\text {TM }}$ technologies), supplemented with penicillin (100 units $/ \mathrm{ml})$, streptomycin $(100 \mu \mathrm{g} / \mathrm{ml})$, epidermal growth factor (murine $\mathrm{EGF}, 20 \mathrm{ng} / \mathrm{ml}$, PEPROtech), fibroblast growth factor (human FGF-basic, $20 \mathrm{ng} /$ $\mathrm{ml}$, PEPROtech), platelet-derived growth factor (PDGF-AB, $20 \mathrm{ng} / \mathrm{ml}$, PEPROtech) and NeuroCult ${ }^{\text {TM }}$ NS-A Proliferation Supplement (Human, STEMCELL ${ }^{\text {TM }}$ technologies). QCTB ROS 006 was obtained from A. Moore, GPC16 cells were established from a patient treated at Great Ormond Street Hospital diagnosed with high-grade glioma. All patient samples were collected under full Research Ethics Committee approval. The pediatric glioma cell lines were obtained from C. Jones and were cultured in Dulbecco's Modified Eagle's Medium/Nutrient Mixture F-12 (DMEM/F-12, SIGMA Life Science) supplemented with penicillin $(100 \mathrm{units} / \mathrm{ml})$, streptomycin $(100 \mu \mathrm{g} / \mathrm{ml})$, and $10 \%$ Fetal Bovine Serum (FBS, Gibco by Life Technologies). All cells were grown as monolayers at $37^{\circ} \mathrm{C}$ in $5 \% \mathrm{CO}_{2}$. All cells were regularly tested for mycoplasma and purity of the culture by STR profiling.

\section{Drug treatment}

Prior to their use, disulfiram (Sigma-Aldrich) and auranofin (Tocris) were dissolved in DMSO. The dissolved drugs were added to the cells for treatment, the same quantity of DMSO (without drug) was added to the control samples. $\mathrm{CuCl}_{2}$ was added to all samples of experiments involving disulfiram (also to the control sample) to reach a final concentration of $1 \mu \mathrm{M}$.

\section{Cell viability assay}

The CellTiter-Glo Luminescent Cell Viability Assay (Promega) was used according to the manufacturer's protocol. Cells were lysed on an orbital shaker for $2 \mathrm{~min}$. After stabilizing the luminescent signal for $10 \mathrm{~min}$ at room temperature, the content of the wells was transferred to a white v-bottom Greiner Bio-One 96-well. Luminescence was measured using a TECAN infinite M200PRO plate reader.

\section{Mitochondrial membrane potential assay}

JC-10 Mitochondrial Membrane Potential Assay (Abcam, ab112134) was used according to the manufacturer's protocol. Luminescence was measured using a TECAN infinite M200PRO plate reader.

\section{Caspase-3 activity assay}

Caspase-3 Activity Assay kit (Abcam, ab252897) was used according to the manufacturer's protocol. Luminescence was measured using a TECAN infinite M200PRO plate reader.

\section{Histone extraction for western blot analysis}

Histones were extracted by adapting the histone extraction protocol for western blot from Abcam. Cells were resuspended in Triton Extraction Buffer (TEB) and lysed for $10 \mathrm{~min}$ at $4^{\circ} \mathrm{C}$. The lysate was centrifuged for $10 \mathrm{~min}$ at $6500 \times g$ at $4{ }^{\circ} \mathrm{C}$ to collect the nuclei. The supernatant was discarded, and the pellet was washed in TEB and centrifuged again using the same conditions. The pellet was resuspended in $0.2 \mathrm{M} \mathrm{HCl}\left(4 \times 10^{7}\right.$ nuclei $\left./ \mathrm{ml}\right)$ and the histones were extracted overnight at $4^{\circ} \mathrm{C}$. The sample was centrifuged for $10 \mathrm{~min}$ at $6500 \times g$ at $4{ }^{\circ} \mathrm{C}$ and the supernatant was collected. $2 \mathrm{M} \mathrm{NaOH}$ was used to neutralize the supernatant. Reducing Sample Buffer was added to the extracts (1:1). The extracts were stored at $-20^{\circ} \mathrm{C}$.

\section{Western blot}

Proteins were resolved on sodium dodecyl sulphate-polyacrylamide gel electrophoresis and transferred to nitrocellulose membrane. Membranes were blocked for one hour in PBS 5\% nonfat dry milk at room temperature and primary antibodies incubated $\mathrm{O} / \mathrm{N}$ at $4{ }^{\circ} \mathrm{C}$. The primary antibodies were detected by DyLight 680 or 800 conjugated secondary antibodies (LI-COR) diluted 1:5000 in PBS Tween $0.1 \%$ and $5 \%$ nonfat dry milk and subsequently visualized with the Odyssey ${ }^{\circledR}$ Infrared Imaging System (LICOR). The antibodies specific for MLL1 (Millipore, 05-764), Vincullin (Abcam, ab129002), H3K4me2 (Abcam, ab32356), H3K4me3 (Abcam, ab8580), H3K9me2/3 (Cell Signalling, 5327), H3 (Abcam, ab10799), MYC (Abcam, ab32072), PAX2 (Abcam, ab79389) SOX2 (Abcam, ab133337) were diluted $1: 1,000$ in PBS Tween $0.1 \%$ and $5 \%$ nonfat dry milk O/N at $4{ }^{\circ} \mathrm{C}$.

RNA isolation, RT-PCR, and quantitative real-time PCR analysis Total RNA was prepared using an RNeasy kit (Qiagen). For reverse transcription, complementary DNA (cDNA) Reverse Transcription Kit (Applied Biosystems) was used and quantitative real-time PCR was performed using Maxima SYBR Green/ROX qPCR Master mix (ThermoFisher Scientific). Relative abundance of the specific mRNAs was normalized to Beta-Actin mRNA. Primer sequences: ACTB: tccgtgtggatcggcggctcca, ctgcttgctgatccacatctg. BAHCC1: catcatcacatccgaaccag, tgaggtggatggatcatttg. HOXA7: ctggatgcggtcttcagg, ggtagcggttgaagtggaac. HOXA9: 
gcgccttctctgaaaacaat, cgctttttccgagtggag. HOXA11: cggcagcagaggagaaag, gtataggggcagcgctttt. HOXA13: cctctggaagtccactctgc, ggtataaggcacgcgcttc. HOXC10: aggagagggccaaagctg, agccaatttcctgtggtgtt. LDHA: ccgttacctaatgggggaaa, gcaacattcattccactcca MLL1: gtcgaccgttgccttctg, gggtgataaggaagaggtactgtg. MLL2: ggagaaccagaccattgtgc, ttctgaatgggcgagtgg. MYC: gaccagctggagatggtgac, ggtcgcagatgaaactctgg. OLIG2: tcctcaaatcgcatccaga, gaaaaaggtcatcgggctct. PAX2: cagcgtctcttccatcaacag, gtgctgggaacaatggtgt SOX2: ttgctgcctctttaagactagga, taagcctggggctcaaact.

\section{Chromatin Immunoprecipitation}

In all, $0.5 \times 10^{6}$ cells were crosslinked in $1 \%$ formaldehyde. Chromatin immunoprecipitation was performed using Imprint Chromatin Immunoprecipitation Kit (Sigma-Aldrich, \#CHP1) per manufacturer's instructions. Sonication was performed using Diagenode bioruptor with the following setup: $30 \mathrm{~s}$ on $50 \%$ power, $30 \mathrm{~s}$ off, 10 cycles. For quantitative ChIP, DNA amplification was performed with Maxima SYBR Green/ROX qPCR Master mix (ThermoFisher Scientific). Percent input was calculated with the formula $100 \times 2^{\text {(Ctadjusted input }}$

-CtIP). Input DNA Ct was adjusted from $1 \%$ to $100 \%$ equivalent by subtracting $6.644 \mathrm{Cts}\left(\log _{2} 100\right)$ from original Ctinput. Two micrograms of MLL1 (Bethyl, A700-010) or H3K4me2 (Abcam, ab32356) antibodies were used. Primers sequence are MYC promoter: actcacaggacaaggatgcg, gcgcgcctaccattttcttt. PAX2 promoter: caagtcatccatctcccggc, tcccggtgtgtgtctctcta. GAPDH promoter: caattccccatctcagtcgt, tagtagccgggecctacttt.

\section{MLL1 and MLL2 downregulation}

Small interfering RNAs (siRNA) were purchased from Dharmacon (MLL1: ONTARGETplus SMART pool Human MLL, MLL2: ON-TARGETplus SMART pool Human MLL4). ON-TARGETplus Control pool Non-Targeting pool was used for control. Cells were plated and transfected on the following day using OPTI-MEM (gibco by Life Technologies) and Lipofectamine RNAiMAX (Invitrogen) according to the manufacturer's instructions. Nine microliters Lipofectamine was added to $150 \mu \mathrm{l}$ OPTI-MEM. The appropriate amount of siRNA (30 pmol for SF188, 300 pmol for GPC16 and QCTB R006) was diluted in $150 \mu \mathrm{l}$ OPTI-MEM. The two solutions prepared were mixed and incubated for $5 \mathrm{~min}$. Two-hundred fifty microliters of this mix was added to the cells.

\section{RNA-seq}

Library preparation, RNA sequencing, and reads alignment were conducted by the UCL genomics team. RNA integrity was confirmed using the Agilent 2200 TapeStation System (Agilent technologies). Two-hundred nanograms of total RNA were processed using the NEBNext RNA Ultra II kit with Poly A + selection (p/n E7760 \& E7490) according to manufacturer's instructions. Oligo dT beads were used to pull down polyadenylated mRNA transcripts and isolate them from total RNA. Chemical fragmentation was applied to the purified mRNA. The fragments were primed with random hexamers, and strand-specific first-strand cDNA was generated 80 using Reverse Transcriptase and Actinomycin-D. dUTP instead of dTTP was used for the synthesis of the second CDNA strand to mark it. To prevent selfligation and adapter dimerization the CDNA was then "A-tailed" at the 3' end. Truncated $Y$ adaptors with a $T$ overhang were ligated to the A-Tailed cDNA. The ligated fragments were amplified with 14 cycles of PCR. The primers used contain a $6 \mathrm{bp}$ Index sequence that allows each library to be identified. Only the first-strand CDNA is amplified since the taq polymerase employed in the PCR is unable to read through uracil. This makes the library strand-specific. Qubit and Bioanalyser fragment analyses were used to calculate equimolar quantities for the libraries to be multiplexed in the same run. Sequencing was performed on the NextSeq 500 instrument (Illumina, San Diego, US) using a 43 bp paired-end run. This resulted in $>$ 15 million reads per sample. Illumina's bcl2fastq Conversion Software v2.19 was used to demultiplex and convert the run data to fastq files. These were aligned to the human genome UCSC hg38 using RNA-STAR 2.5.2b and deduplicated using Picard Tools 1.79. FeatureCounts was used to count the reads per transcript and differential expression assessed with the BioConductor package SARTools, a DESeq2 wrapper. The annotation and sequences were obtained from Illumina iGenomes. RNA-seq data can be accessed via GEO Profiles (GSE139016).

\section{Gene set enrichment analysis (GSEA)}

RNAseq data sets consisted of all genes with a mean count above 10 in the MLL1/MLL2 knockdown or disulfiram-treated sample and the control sample. The data sets were analyzed for the enrichment of gene sets using the Gene Set Enrichment Analysis (GSEA) software from the Broad Institute. Each gene set included between 15 and 500 genes and can be found in the Molecular
Signature Database (MSigDB) or in appendix 1. The following settings were applied for the GSEA: numbers of permutations: 1000; permutation type: gene set. A gene set was considered significantly enriched if the nominal $p$ value 81 was $<0.05$. According to GSEA results with a false-discovery rate (FDR) below $25 \%$ can indicate a trend. Thus, results with a p-value above 0.05 and an FDR below $25 \%$ were considered as potential findings. Correlation between data sets was detectable as a positive enrichment score, while inverse correlation showed as a negative score.

\section{Statistical analysis}

Statistical analyses were performed using graphpad Prism software.

\section{REFERENCES}

1. Yoo $C B$, Jones PA. Epigenetic therapy of cancer: past, present and future. Nat Rev Drug Discov. 2006;5:37-50.

2. Schuettengruber B, Bourbon H-M, Di Croce L, Cavalli G. Genome regulation by polycomb and trithorax: 70 years and counting. Cell 2017;171:34-57.

3. Gallo M, Ho J, Coutinho FJ, Vanner R, Lee L, Head R, et al. A tumorigenic MLLhomeobox network in human glioblastoma stem cells. Cancer Res. 2013;73:417.

4. Heddleston JM, Wu Q, Rivera M, Minhas S, Lathia JD, Sloan AE, et al. Hypoxiainduced mixed-lineage leukemia 1 regulates glioma stem cell tumorigenic potential. Cell Death Differ. 2012;19:428-39.

5. Parsons DW, Li M, Zhang X, Jones S, Leary RJ, Lin JC, et al. The genetic landscape of the childhood cancer medulloblastoma. Science. 2011;331:435-9.

6. Costa BM, Smith JS, Chen Y, Chen J, Phillips HS, Aldape KD, et al. Reversing HOXA9 oncogene activation by PI3K inhibition: epigenetic mechanism and prognostic significance in human glioblastoma. Cancer Res. 2010;70:453-62.

7. Duan R, Han L, Wang Q, Wei J, Chen L, Zhang J, et al. HOXA13 is a potential GBM diagnostic marker and promotes glioma invasion by activating the Wnt and TGF$\beta$ pathways. Oncotarget. 2015;6:27778-93.

8. Tabuse M, Ohta S, Ohashi $Y$, Fukaya $R$, Misawa A, Yoshida $K$, et al. Functional analysis of HOXD9 in human gliomas and glioma cancer stem cells. Mol Cancer. 2011;10:60.

9. Gaspar N, Marshall L, Perryman L, Bax DA, Little SE, Viana-Pereira M, et al. MGMTindependent temozolomide resistance in pediatric glioblastoma cells associated with a PI3-kinase-mediated HOX/stem cell gene signature. Cancer Res. 2010;70:9243.

10. Kola I, Landis J. Can the pharmaceutical industry reduce attrition rates? Nat Rev Drug Discov. 2004;3:711-6.

11. Sleire L, Førde $H E$, Netland $I A$, Leiss $L$, Skeie BS, Enger $P \varnothing$. Drug repurposing in cancer. Pharmacol Res. 2017;124:74-91.

12. Ekinci E, Rohondia S, Khan R, Dou QP. Repurposing disulfiram as an anti-cancer agent: updated review on literature and patents. Recent Pat Anticancer Drug Discov. 2019;14:113-32.

13. Skrott Z, Mistrik M, Andersen KK, Friis S, Majera D, Gursky J, et al. Alcohol-abuse drug disulfiram targets cancer via p97 segregase adaptor NPL4. Nature. 2017:552:194-9.

14. Bax DA, Little SE, Gaspar N, Perryman L, Marshall L, Viana-Pereira M, et al. Molecular and phenotypic characterisation of paediatric glioma cell lines as models for preclinical drug development. PLoS ONE. 2009;4:e5209.

15. Wang $P$, Lin C, Smith ER, Guo H, Sanderson BW, Wu M, et al. Global analysis of H3K4 methylation defines MLL family member targets and points to a role for MLL1-mediated H3K4 methylation in the regulation of transcriptional initiation by RNA polymerase II. Mol Cell Biol. 2009;29:6074-85.

16. Glaser S. Multiple epigenetic maintenance factors implicated by the loss of MII2 in mouse development. Development. 2006;133:1423-32.

17. Xu W, Su C-H, Lin IH, Tzeng T-Y, Hsieh W-T, Hsu M-T. Regulation of IL-20 expression by estradiol through KMT2B-mediated epigenetic modification. PLoS ONE. 2016;11:e0166090

18. Kerimoglu C, Agis-Balboa RC, Kranz A, Stilling R, Bahari-Javan S, Benito-Garagorri $E$, et al. Histone-methyltransferase MLL2 (KMT2B) is required for memory formation in mice. J Neurosci. 2013;33:3452-64.

19. Bhagwat AS, Vakoc CR. Targeting transcription factors in cancer. Trends Cancer 2015;1:53-65

20. Rheinbay E, Suvà Mario L, Gillespie Shawn M, Wakimoto $H$, Patel Anoop $P$, Shahid $M$, et al. An aberrant transcription factor network essential for wnt signaling and stem cell maintenance in glioblastoma. Cell Rep. 2013;3:1567-79.

21. Ligon KL, Alberta JA, Kho AT, Weiss J, Kwaan MR, Nutt $C L$, et al. The oligodendroglial lineage marker OLIG2 is universally expressed in diffuse gliomas. J Neuropathol Exp Neurol. 2004;63:499-509.

22. Lu F, Chen Y, Zhao C, Wang H, He D, Xu L, et al. Olig2-dependent reciprocal shift in PDGF and EGF receptor signaling regulates tumor phenotype and mitotic growth in malignant glioma. Cancer Cell. 2016;29:669-83. 
23. Annovazzi L, Mellai M, Caldera V, Valente G, Schiffer D. SOX2 expression and amplification in gliomas and glioma cell lines. Cancer Genomics Proteom. 2011;8:139-47.

24. Eilers M, Eisenman RN. Myc's broad reach. Genes Dev. 2008;22:2755-66.

25. Blake JA, Ziman MR. Pax genes: regulators of lineage specification and progenitor cell maintenance. Development. 2014;141:737-51.

26. Uhlen M, Zhang C, Lee S, Sjostedt E, Fagerberg L, Bidkhori G, et al. A pathology atlas of the human cancer transcriptome. Science. 2017;357:eaan2507. https:// doi.org/10.1126/science.aan2507.

27. Cvek B. Nonprofit drugs as the salvation of the world's healthcare systems: the case of Antabuse (disulfiram). Drug Discov Today. 2012;17:409-12.

28. Hothi P, Martins TJ, Chen L, Deleyrolle L, Yoon J-G, Reynolds B, et al. Highthroughput chemical screens identify disulfiram as an inhibitor of human glioblastoma stem cells. Oncotarget. 2012;3:1124-36.

29. Triscott J, Lee $C$, Hu K, Fotovati A, Berns R, Pambid M, et al. Disulfiram, a drug widely used to control alcoholism, suppresses the self-renewal of glioblastoma and over-rides resistance to temozolomide. Oncotarget. 2012;3:1112-23.

30. Lun X, Wells JC, Grinshtein N, King JC, Hao X, Dang N-H, et al. Disulfiram when combined with copper enhances the therapeutic effects of temozolomide for the treatment of glioblastoma. Clin Cancer Res. 2016;22:3860.

31. Cantilena S, Gasparoli L, Pal D, Heidenreich O, Klusmann J-H, Martens JHA, et al. Non-toxic therapeutic direct targeting of MLL-fusion proteins using a rational drug screening approach (paper under submission). 2020.

32. Veverka KA, Johnson KL, Mays DC, Lipsky JJ, Naylor S. Inhibition of aldehyde dehydrogenase by disulfiram and its metabolite methyl diethylthiocarbamoylsulfoxide. Biochemical Pharmacol. 1997;53:511-8.

33. Long Hannah K, Blackledge Neil P, Klose Robert J. ZF-CxxC domain-containing proteins, CpG islands and the chromatin connection. Biochemical Soc Trans. 2013;41:727-40.

34. Biaglow JE, Miller RA. The thioredoxin reductase/thioredoxin system: Novel redox targets for cancer therapy. Cancer Biol Ther. 2014;4:13-20.

35. Papaioannou M, Mylonas I, Kast RE, Brüning A. Disulfiram/copper causes redoxrelated proteotoxicity and concomitant heat shock response in ovarian cancer cells that is augmented by auranofin-mediated thioredoxin inhibition. Oncoscience. 2013;1:21-9.

36. Rao RC, Dou Y. Hijacked in cancer: the KMT2 (MLL) family of methyltransferases. Nat Rev Cancer. 2015;15:334-46.

37. Barski A, Cuddapah S, Cui K, Roh T-Y, Schones DE, Wang Z, et al. Highresolution profiling of histone methylations in the human genome. Cell. 2007;129:823-37.

38. Liu P, Brown S, Goktug T, Channathodiyil $P$, Kannappan V, Hugnot JP, et al. Cytotoxic effect of disulfiram/copper on human glioblastoma cell lines and ALDH-positive cancer-stem-like cells. Br J Cancer. 2012;107:1488-97.

39. Ansari KI, Kasiri S, Mandal SS. Histone methylase MLL1 has critical roles in tumor growth and angiogenesis and its knockdown suppresses tumor growth in vivo. Oncogene. 2012;32:3359-70.

40. Zhang C, Song C, Liu T, Tang R, Chen M, Gao F, et al. KMT2A promotes melanoma cell growth by targeting hTERT signaling pathway. Cell Death Dis. 2017;8: e2940-e.

41. Lu C, Paschall AV, Shi H, Savage N, Waller JL, Sabbatini ME, et al. The MLL1H3K4me3 axis-mediated PD-L1 expression and pancreatic cancer immune evasion. J Natl Cancer Inst. 2017;109:djw283.

42. Ansari Kl, Kasiri S, Mandal SS. Histone methylase MLL1 has critical roles in tumor growth and angiogenesis and its knockdown suppresses tumor growth in vivo. Oncogene. 2013;32:3359-70.

\section{ACKNOWLEDGEMENTS}

The authors thank Tony Brooks and Dr. Paola Niola from UCL GOS ICH Genomic Facility for providing assistance with conducting the RNA-seq experiments. We thank Dr. Chris Jones for providing the cell lines and the patient primary cells QCTB R006. This research was funded by grants from the Brain Tumor Charity (BTC 8/197) and The Olivia Hodson Cancer Fund (SR16A26) and supported by the National Institute for Health Research Biomedical Research Centre at Great Ormond Street Hospital for Children NHS Foundation Trust and University College London.

\section{AUTHOR CONTRIBUTIONS}

S.M., S.C., D.M., and J.d.B. designed the experiments. S.M., D.M., and J.d.B. performed the experiments and data analysis. J.A. and D.M. prepared the GPC16 primary cells. D. M. and J.d.D. supervised the study. S.M. and D.M. wrote the manuscript. S.C., M.V.N.C., J.A., D.H., and P.S. provided critical input on the manuscript. All authors reviewed and approved the final manuscript.

\section{ETHICS STATEMENT}

This study has been approved by the Health Research Authority, UK. Project ID: 88584

\section{COMPETING INTERESTS}

The authors declare no competing interests.

\section{ADDITIONAL INFORMATION}

Supplementary information The online version contains supplementary material available at https://doi.org/10.1038/s41419-021-04078-9.

Correspondence and requests for materials should be addressed to J.d.B. or D.M.

Reprints and permission information is available at http://www.nature.com/ reprints

Publisher's note Springer Nature remains neutral with regard to jurisdictional claims in published maps and institutional affiliations.

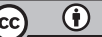

Open Access This article is licensed under a Creative Commons Attribution 4.0 International License, which permits use, sharing, adaptation, distribution and reproduction in any medium or format, as long as you give appropriate credit to the original author(s) and the source, provide a link to the Creative Commons license, and indicate if changes were made. The images or other third party material in this article are included in the article's Creative Commons license, unless indicated otherwise in a credit line to the material. If material is not included in the article's Creative Commons license and your intended use is not permitted by statutory regulation or exceeds the permitted use, you will need to obtain permission directly from the copyright holder. To view a copy of this license, visit http://creativecommons. org/licenses/by/4.0/.

(c) The Author(s) 2021 